\title{
Macroscopic Quantum Tunneling in "Small" Josephson Junctions in magnetic field
}

\author{
Yu.N.Ovchinnikov, ${ }^{1}$ A. Barone ${ }^{2}$ and A.A.Varlamov ${ }^{3}$ \\ ${ }^{1}$ Max-Plank Institute for Physics of Complex Systems, Dresden, \\ D-01187 Germany and Landau Institute for Theoretical Physics, \\ RAS, Chernogolovka, Moscow District, 142432 Russia \\ ${ }^{2}$ Dipartimento Scienze Fisiche, Universita' di Napoli "Federico II" \\ and Coherentia-INFM, CNR, piazzale Tecchio, 80, 80125, Napoli, Italy \\ ${ }^{3}$ Coherentia-INFM, CNR, Viale del Politecnico 1, \\ I-00133 Rome, Italy and Materials Science Division, \\ Argonne National Laboratory, 9700 S.Cass Avenue, Argonne Il 60439, USA
}

(Dated: October 3, 2018)

\begin{abstract}
We study the phenomenon of macroscopic quantum tunneling (MQT) in small Josephson junctions (JJ) with an externally applied magnetic field. The latter results in the appearance of the Fraunhofer type modulation of the current density along the barrier. The problem of MQT for a point-like JJ is reduced to the motion of the quantum particle in the washboard potential. In the case of a finite size JJ under consideration, this problem corresponds to a MQT in potential which itself, besides the phase, depends on space variables. Finally, the general expression for the crossover temperature $T_{0}$ between thermally activated and macroscopic quantum tunneling regimes and the escaping time $\tau_{\text {esc }}$ have been calculated.

PACS numbers: $74.50 .+\mathrm{r}$
\end{abstract}

The paramount importance of macroscopic quantum tunneling lies in the role of such a process in a variety of physical phenomena, which span from the cosmological implications of the false "vacuum" to recent studies on BEC systems. Great attention deserves in this context the occurrence of MQT in Josephson junctions $\frac{1}{\underline{\underline{m}}}$ observed both in low $T_{c^{2}}$ and high $T_{c} \frac{3}{3}$ structures. Usually the phenomenon of MQT is considered in a "small" JJ, i.e. in the assumption that the characteristic size $L$ of the junction is much smaller than all other related parameters of the problem (some exceptions can be found in experimenta $\underline{1}^{\underline{4}}$ and theoretical papers ${ }^{\underline{5}}$ ). Such parameters are the Josephson penetration depth $\lambda_{J}=\left(\hbar c^{2} / 8 \pi e j_{1} \ell_{\text {eff }}\right)^{1 / 2}$ and the characteristic length $L_{H}=\ell_{H}^{2} / \ell_{\text {eff }}$, with $\ell_{H}=\left(\hbar c / e H_{\text {ext }}\right)^{1 / 2}$ as the standard quantum mechanical magnetic length. The external magnetic field $H_{\text {ext }}$ is supposed to be directed along the plane of junction. The critical current density $j_{1}$ of the JJ will be defined explicitly below. The effective length $\ell_{\text {eff }}$ depends on the relation between the thickness $d_{(i)}(i=L, R)$ of the superconductive electrodes and the magnetic penetration depth $\lambda_{(i)}$ of the bulk superconductor materials, which the electrodes are made of. In the limiting cases one can find $\underline{6,7,8,9}$

$$
\ell_{\mathrm{eff}}=\left\{\begin{array}{l}
\lambda_{(1)}+\lambda_{(2)}+d_{\mathrm{ox}}, \lambda_{(i)} \ll d_{(i)} \\
d_{(1)}+d_{(2)}+d_{\mathrm{ox}}, d_{(i)} \ll \lambda_{(i)}
\end{array}\right.
$$

The finite value of the junction length $L$ becomes important even for $L<\lambda_{J}$. Such a situation takes place when the magnetic field is applied to the junction what results in appearance of the Fraunhofer diffraction pattern $I_{c}$ vs $H_{\text {ext }}$. In our analysis we will suppose that $L \ll \lambda_{J}$ but will not impose any restrictions on the relation between $L$ and $L_{H}$.

The effect of magnetic field on the properties of JJ leads to three different manifestations. First of all magnetic applied filed suppresses the value of the order parameter $\Delta$ and changes the frequency dependence of the singleparticle density of states $\rho(\omega)$ (formally, the presence of magnetic field changes the frequency dependence of the Green functions $)^{10}$. Then, its presence leads to the spacial modulation of the current flowing through the junction $\underline{6}, 7,8,9,11$. Finally, the magnetic field affects on the probability of the electron tunneling through the barrier. Since the thickness of insulating barriers in the case of JJ is very small (about $10 \AA$ ) this effect we will neglect in our discussion.

Let us start our analysis from the suppression of the order parameter $\Delta$ and changes of the frequency dependence of the Green functions by the magnetic field. The Green functions integrated over the energy variable $(\alpha, \beta)$ for both electrodes can be found from the equation 10 :

$$
\alpha \Delta-\beta \omega+\frac{D}{2}\left\{\alpha\left(\frac{\partial}{\partial \mathbf{r}}-2 i e \mathbf{A}\right)^{2} \beta-\beta \frac{\partial^{2}}{\partial \mathbf{r}^{2}} \alpha\right\}=\alpha \beta \Gamma,
$$

with the normalization condition $\alpha^{2}(\mathbf{r}, \omega)+\beta^{2}(\mathbf{r}, \omega)=1$. We choose the vector potential gauge in each electrode in the form $\mathbf{A}=(H z, 0,0)$, where the direction of $\widehat{z}$ axis is supposed to be perpendicular to the junction surface and the magnetic field $\mathbf{H}$ is directed along the $\widehat{y}$-axis. The coordinate $z$ in each electrode changes in the symmetric interval $z \in[-d / 2, d / 2]$, while the coordinate along the barrier $x \in[-L / 2, L / 2]$. In view of the smallness of the JJ length one 
can see that the spacial dependence of $\Delta$ and Green functions can be neglected. Averaging the Eq.(2) over the space variable one can determine the frequency dependence of the averaged Green functions $(\bar{\alpha}(\omega), \bar{\beta}(\omega))$

$$
\bar{\alpha}_{(i)} \Delta_{(i)}-\bar{\beta}_{(i)} \omega=\bar{\alpha}_{(i)} \bar{\beta}_{(i)}\left(\Gamma_{(i)}+D_{(i)} e^{2} H_{\mathrm{ext}}^{2} d_{(i)}^{2} / 6\right)
$$

where $\Gamma_{(i)}=\tau_{s(i)}^{-1}$ is the inverse electron spin-flip scattering time.

The critical current density $J_{1}(H)$ can be determined from the Ambegaokar-Baratoff type formula 11 :

$$
J_{1}(H)=\frac{\pi}{e R_{\mathrm{N}} S} T \sum_{\omega} \bar{\beta}_{(1)}(\omega) \bar{\beta}_{(2)}(-\omega), \quad \omega \equiv \omega_{n}=2 \pi T\left(n+\frac{1}{2}\right),
$$

where $R_{N}$ is the junction resistance in normal state and $S$ is the junction area. Thus the effect of magnetic field results just in the renormalization of the critical current density. The latter becomes essential when $D_{(i)} e^{2} H_{\mathrm{ext}}^{2} d_{(i)}^{2} \sim \Delta$.

Let us discuss of the second external magnetic field manifestation on the properties of a JJ, related to the space modulation of current density in it. In the case of a "point-like" JJ the value $J_{1}$ determines the height of the potential barriers and hence the escaping time for the MQT. In the case of a small JJ of finite size the current density is space modulated by the external magnetic field and the escaping time calculation becomes more complicated. Indeed, while for the point contact it is reduced to the motion of the quantum particle in the washboard potential in the case under consideration the problem corresponds to the tunneling of a lengthy object in the potential which itself, besides the phase, depends on space variables.

To calculate the escaping time $\tau_{\text {esc }}$ we need to write down the functional of effective action $A[\varphi]$, what can be done in this case basing on the results of ${ }^{12,13}$ :

$$
\begin{aligned}
A[\varphi] & =\frac{1}{S} \int_{-1 / 2 T}^{1 / 2 T} d \tau \int d^{2} \mathbf{r}\left\{\frac{C}{2 e^{2}}\left(\frac{\partial \varphi(\mathbf{r}, \tau)}{\partial \tau}\right)^{2}-\frac{\hbar}{e} I \varphi(\mathbf{r}, \tau)-\frac{\pi \hbar}{2 R_{\mathrm{N}} e^{2}} \int_{-1 / 2 T}^{1 / 2 T} d \tau_{1}\left[2 \sin ^{2}\left(\frac{\varphi(\mathbf{r}, \tau)-\varphi\left(\mathbf{r}, \tau_{1}\right)}{2}\right)\right.\right. \\
& \left.\times \alpha_{L}\left(\tau-\tau_{1}\right) \alpha_{R}\left(\tau-\tau_{1}\right)+\cos \left(\varphi(\mathbf{r}, \tau)+\varphi\left(\mathbf{r}, \tau_{1}\right)\right) \beta_{L}\left(\tau-\tau_{1}\right) \beta_{R}\left(\tau-\tau_{1}\right)\right] \\
& \left.+\frac{\pi \hbar T^{2}}{R_{\mathrm{sh}} e^{2}} \int_{-1 / 2 T}^{1 / 2 T} d \tau_{1} \frac{\sin ^{2}\left(\frac{\varphi(\mathbf{r}, \tau)-\varphi\left(\mathbf{r}, \tau_{1}\right)}{2}\right)}{\sin ^{2}\left[\pi T\left(\tau-\tau_{1}\right)\right]}+\frac{\hbar^{2} c^{2} S}{8 \pi e^{2} \ell_{\mathrm{eff}}}\left(\frac{\partial \varphi(\mathbf{r}, \tau)}{\partial \mathbf{r}}-\frac{e \ell_{\mathrm{eff}}}{\hbar c}\left[\mathbf{H}_{\mathrm{ext}} \times \mathbf{n}\right]\right)^{2}\right\} .
\end{aligned}
$$

Here $C$ is the junction capacity, $I$ is the full current flowing through the junction, $R_{\mathrm{sh}}$ is the shunt resistance. The integrals are carried out over the imaginary time and the junction area. The first term corresponds to the kinetic energy of the junction. The second and fourth terms describe the contribution of the potential energy to the action. Let us stress that the third term, corresponding to the capacity renormalization appears only beyond the quasi-classical approximation. The action accounting for all these terms was already found in ${ }^{12,13}$ in the case of microscopic study of the Josephson current decay in point-like junction. The last term in Eq.(5) accounts for the finite size of the junction and the magnetic field contribution to the effective action. The motion in the classically permitted region is described by the same functional Eq.(5) in the assumption $\tau=\tau_{1}$ what eliminates the third term. The further variation procedure of Eq.( 5) over $\varphi$ results in the getting of the quasi-classical equations of motion $6.7,8,9$.

We are looking for the escape time $\tau_{\text {esc }}$ with the accuracy up to the pre-exponential factor. The exponential factor in it is determined by the extremal value of the functional Eq.(5) found at the class of solutions periodical in imaginary time $\tau$ with the period $1 / T$. The function $\varphi(x, \tau)$ at which the extremum of functional Eq.(5) is realized (the saddle point of functional ) we will search in the form

$$
\varphi(x, \tau)=\varphi_{0}-\frac{x}{L_{H}}+\widetilde{\varphi}(x, \tau)=\frac{1}{2} \Phi(x)+\widetilde{\varphi}(x, \tau),
$$

where $\varphi_{0}=$ const, is the phase difference at the junction in the absence of magnetic field, while the function $\widetilde{\varphi}(x, \tau)$ satisfies the boundary condition $\underline{\underline{9}}$

$$
\left.\frac{\partial \widetilde{\varphi}}{\partial x}\right|_{x=-L / 2}=\left.\frac{\partial \widetilde{\varphi}}{\partial x}\right|_{x=L / 2}=0 .
$$

The experimentally realistic case corresponds to the current close to its critical value $I_{c}$. This is why we restrict our consideration namely by the condition $I \rightarrow I_{c}$. Let us note that in this case the third term in Eq. ( 5 ) can be 
excluded from consideration by the simple renormalization of the junction capacity $C \rightarrow C^{*}$, where

$$
C^{*}=C+\frac{\pi \hbar}{2 R_{\mathrm{N}}} \int_{-\infty}^{\infty} \frac{d \omega}{2 \pi}\left(\frac{\partial \alpha_{L}}{\partial \omega}\right)\left(\frac{\partial \alpha_{R}}{\partial \omega}\right)
$$

The substitution of Eq.( (6) to the Eq. (5) with the further expansion of the cosine over $\widetilde{\varphi}(x, \tau)$ in the fourth term leads to the cancellation of the linear in $\widetilde{\varphi}$ second term. Since the function $\widetilde{\varphi}$ has to be periodical in imaginary time $\tau$ with the period $1 / T$ we present it in the form of the Fourier series. This makes the effective action (5) to become the functional of the Fourier coefficients $b_{n}$ (let us note that $b_{n}(x)=b_{-n}(x)$ ):

$$
\begin{gathered}
A\left[b_{n}\right]=\frac{\hbar J_{1}}{e T} \int d^{2} \mathbf{r} \sum_{n=-\infty}^{\infty}\left\{\left[\cos [\Phi(x)]+\zeta\left(n^{2}+Q_{\operatorname{sh}}|n|\right)\right] b_{n}^{2}\right. \\
\left.-\frac{2}{3} \sin [\Phi(x)]\left(\sum_{k=-\infty}^{\infty} b_{k} b_{k+n}\right) b_{n}+\lambda_{J}^{2}\left(\frac{\partial b_{n}}{\partial x}\right)^{2}\right\},
\end{gathered}
$$

where we have introduced the notations $\zeta=2 \pi^{2} T^{2} C^{*} /\left(e \hbar J_{1} S\right)$ and the Q-factor $Q_{\mathrm{sh}}^{-1}=2 \pi T R_{\mathrm{sh}} C^{*} / \hbar$.The further variation of the functional (9) over $b_{n}$ results in the system of equations:

$$
-\lambda_{J}^{2} \frac{\partial^{2} b_{n}}{\partial x^{2}}+\left[\cos [\Phi(x)]+\zeta\left(n^{2}+Q_{\mathrm{sh}}|n|\right)\right] b_{n}-\sin [\Phi(x)]\left[\left(2-\delta_{n, 0}\right) b_{0} b_{n}+2 \sum_{k=1}^{\infty} b_{k} b_{n+k}+\sum_{k=1}^{n-1} b_{k} b_{n-k}\right]=0
$$

Let us recall that we will look for the solution of this system with the boundary conditions (77). The analogous system of equations for the point junction was solved for the cases of low and high shunt resistance $\frac{12}{2}$ and also close to the temperature $T_{0}$ corresponding to the crossover between the thermally activating (Arrenius law) and quantum regimes in tunneling through the barrier.

In order to calculate the escape time we need to know the value of the effective action (5) at the saddle point, i.e. at the optimal trajectories $b_{n}(x)$, being solutions of the system (10). The related expression can be considerably simplified, subtracting from the functional Eq. (9) the Eq. (10) multiplied by corresponding $b_{n}(x)$ :

$$
\widetilde{A}\left[b_{n}\right]=\frac{\hbar J_{1}}{3 e T} \int d^{2} \mathbf{r} \sin [\Phi(x)]\left[b_{0}^{3}+6 b_{0} \sum_{n=1}^{\infty} b_{n}^{2}+4 \sum_{n=1}^{\infty} b_{n} \sum_{k=1}^{\infty} b_{k} b_{n+k}+2 \sum_{n=1}^{\infty} b_{n} \sum_{k=1}^{n-1} b_{k} b_{n-k}\right] .
$$

We consider the junction of a small enough size: $L \ll \lambda_{J}$. Thus the system Eq. (10) acquires the large parameter $\lambda_{J}^{2} / L^{2} \gg 1$. Consequently the functions $b_{n}(x)$ weakly vary with the change of its argument and can be represented in the form:

$$
b_{n}=\left\langle b_{n}\right\rangle+\widetilde{b}_{n}, \quad\left|\widetilde{b}_{n}\right| \ll\left|\left\langle b_{n}\right\rangle\right|,
$$

where $\langle\ldots\rangle$ indicates the average over the coordinate $x$ along the junction. The corresponding equation for $\widetilde{b}_{n}$ takes the form

$$
\lambda_{J}^{2} \frac{\partial^{2} \widetilde{b}_{n}}{\partial x^{2}}=\left\langle b_{n}\right\rangle \sin \left(2 \varphi_{0}\right) \sin \left(\frac{2 x}{L_{H}}\right) .
$$

Its solution satisfying the boundary conditions ( 7 ) is

$$
\widetilde{b}_{n}=\frac{L_{H}^{2}}{4 \lambda_{J}^{2}}\left\langle b_{n}\right\rangle \sin \left(2 \varphi_{0}\right)\left[\left(\frac{2 x}{L_{H}}\right) \cos \left(\frac{L}{2 L_{H}}\right)-\sin \left(\frac{2 x}{L_{H}}\right)\right] .
$$

What concerns the equations for the average values $\left\langle b_{n}\right\rangle$ they can be obtained directly from the Eq.( 10 ) by the method of subsequent approximations.

Now we can calculate the escape time $\tau_{\text {esc }}$ near the crossover temperature $T_{0}$, taking into account the effects of the current density coordinate dependence, due to the applied magnetic field. All coefficients $\left\langle b_{n}\right\rangle$ with $n \geq 1$ are small and quickly decrease as $n$ increases. It is why at temperatures close to $T_{0}$ in Eq. (11) one can restrict consideration by the contribution of $b_{0}, b_{1}$ and $b_{2}\left(\sim b_{1}^{2}\right)$. 
The crossover temperature $T_{0}$ is characterized by the fact that below it the quantum tunneling through barrier becomes more probable than the thermally activated escape process. Formally this temperature is the bifurcation point of the system (10) when the first non-zero solutions for $n \geq 1$ appear. Above it exists the only, trivial, solution is $b_{0}=$ const, what results in the action $([5]) \widetilde{A}[\widetilde{\varphi}]=\Delta U / T$ corresponding to the Arrenius law for the escape time. Below $T_{0}$ the non-zero solutions $b_{n}$ appear, what leads to the appearance of the imaginary time dependence of $\widetilde{\varphi}$, the action (5) changes its form and,consequently, the temperature dependence of the escape time $\tau_{\operatorname{esc}} \sim \exp \left[-A\left(\varphi_{0}, L\right)\right]$ changes qualitatively. After some cumbersome but straightforward calculations one can find the quadratic equation determining the crossover temperature $T_{0}$ :

$$
\frac{2 \pi^{2} C^{*} T_{0}^{2}}{\hbar e}+\frac{\pi T_{0}}{R_{\mathrm{sh}} e}=I_{c} \sin \left(2 \varphi_{0}\right)\left(\frac{L_{H}}{L}\right) \sin \left(\frac{L}{L_{H}}\right) \Upsilon\left(\varphi_{0}, L\right)
$$

where $I_{c}=J_{1}(H) S$ is the value of junction's critical current, while

$$
\Upsilon\left(\varphi_{0}, L\right)=\cot \left(2 \varphi_{0}\right)+\sin \left(2 \varphi_{0}\right)\left(\frac{L_{H}}{2 \lambda_{J}}\right)^{2}\left[\frac{3}{2} \cos \left(\frac{L}{L_{H}}\right)-\left(\frac{L}{L_{H}}\right) \frac{1+\frac{1}{2} \cos \left(\frac{2 L}{L_{H}}\right)}{\sin \left(\frac{L}{L_{H}}\right)}\right] .
$$

In the case of a point junction the right-hand-side of Eq. ( 15 ) reduces to $\sqrt{I_{c}^{2}-I^{2}}$.

In vicinity of $T_{0}$ the essential contribution to Eq. (11) give only the terms with $b_{0}, b_{1}$ and $b_{2}$. Their average values can be found from Eq.(12)-( 14). We present here only

$$
\left\langle b_{1}\right\rangle^{2}=\frac{\Upsilon\left(\varphi_{0}, L\right) \zeta \sin \left(2 \varphi_{0}\right)\left(\frac{L_{H}}{L}\right) \sin \left(\frac{L}{L_{H}}\right)-\zeta^{2}\left(Q_{\mathrm{sh}}+1\right)}{2 \sin ^{2}\left(2 \varphi_{0}\right)\left(\frac{L_{H}}{L}\right)^{2} \sin ^{2}\left(\frac{L}{L_{H}}\right)\left[\frac{2}{Q_{\mathrm{sh}}+1}-\frac{1}{Q_{\mathrm{sh}}+3}\right]} .
$$

what finally permits to find

$$
\widetilde{A}[\varphi]=\frac{\hbar J_{1} S}{3 e T} \sin \left(2 \varphi_{0}\right)\left(\frac{L_{H}}{L}\right) \sin \left(\frac{L}{L_{H}}\right)\left[\Upsilon^{3}\left(\varphi_{0}, L\right)-\frac{6\left\langle b_{1}\right\rangle^{4}}{\zeta} \sin \left(2 \varphi_{0}\right)\left(\frac{L_{H}}{L}\right) \sin \left(\frac{L}{L_{H}}\right)\left(\frac{2}{Q_{\mathrm{sh}}+1}-\frac{1}{Q_{\mathrm{sh}}+3}\right)\right]
$$

One can see that the effect of magnetic field in a finite size junction, even in the case $L \ll \lambda_{J}$, does not reduce simply to the renormalization of the value of $I_{c}$. Indeed, both characteristic parameters of the problem, $T_{0}$ and the escape time $\tau_{\text {esc }}$, turn out to be the functions of two parameters: $I / I_{c}$ and $L / L_{H}$. Let us stress that when the current $I$ is very close to its critical value $I_{c}$ the approximation (12) for the functions $b_{n}(x)$ becomes incorrect, and this region has to be studied separately. These results are of great interest for the whole scenario of quantum coherence, as for instance in the context of MQT in Josephson structures based on d-wave superconductors 3.4

Yu.N.Ovchinnikov acknowledges the financial support of the grant RFBR-06-01-90507-BNTS-a and Russian Foundation of Basic Research.

1 A.J.Leggett, Suppl. Prog. Theor. Phys. 69, 80 (1980) and references reported therein.

2 For an excellent review see: J.Clarke, A.N.Cleland, M.H.Devoret, D.Esteve, and J.H.Martinis, Science 29, 992 (1988).

3 T.Bauch, F.Lombardi, F.Tafuri, A.Barone, G.Rotoli, P.Delsing and T.Claeson, Phys. Rev. Lett. 94, 87003 (2005); T.Bauch, T.Lindstrom, F.Tafuri, G.Rotoli, P.Delsing, T.Claeson and F.Lombardi, Science 311, 57 (2006).

4 A.L.Fetter, M.J.Stephen, Phys. Rev. 168, 475 (1968).

5 M.G. Castellano, G. Torrioli, C. Cosmelli, A. Costantini, F. Chiarello, P. Carelli, G. Rotoli, M. Cirillo and R. L. Kautz Phys. Rev. B 54, 15417 (1996); A. Walraff, A. Lukashenko, J. Lisenfeld, A. Kemp, M.V. Fistul, Y. Koval and A. V. Ustinov, Nature 425, 155 ( 2003); B.Ivlev arXiv: quant-ph/0511052v (7 Nov 2005);

${ }^{6}$ R.E.Eck, D.J.Scalapino, B.N.Taylor, Phys. Rev. Lett. 13, 15 (1964).

7 I.O.Kulik, Soviet Physics - JETP Letters 2, 84 (1965).

8 Yu.M.Ivanchenko, A.V.Svidzinskii and V.A.Slyusarev, Soviet Physics - JETP 24, 131 (1967).

9 A.I.Larkin, Yu.N.Ovchinnikov, Soviet Physics - JETP 26, 1219 (1968).

10 A.I.Larkin, Yu.N.Ovchinnikov, Soviet Physics - JETP 34, 1144 (1972).

11 A.Barone, G.Paterno, Physics and Applications of the Josephson Effect, John Willey, NY (1982).

12 A.I.Larkin, Yu.N.Ovchinnikov, Soviet Physics - JETP 57, 876 (1983).

13 A.I.Larkin, Yu.N.Ovchinnikov, Phys. Rev. B 28, 6281 (1983). 